\title{
Incidence-Polytopes with Toroidal Cells
}

\author{
Asia Ivić Weiss* \\ Department of Mathematics, York University, North York, \\ Ontario, Canada, M3J $1 \mathrm{P3}$
}

Dedicated to H.S. M. Coxeter on the occasion of his 80 th birthday.

\begin{abstract}
A type of partially ordered structures called incidence-polytopes generalizes the notion of polyhedra in a combinatorial sense. The concept includes all regular polytopes as well as many well-known configurations. We use hyperbolic geometry to derive certain types of incidence-polytopes whose cells are isomorphic to maps of type $\{4,4\},\{6,3\}$, or $\{3,6\}$ on a torus. For these structures we give a criterion on the finiteness in terms of groups of $2 \times 2$ matrices, leading among other things to the explicit recognition of the groups in some interesting special cases.
\end{abstract}

\section{Introduction}

Because of their symmetry properties regular polytopes have been investigated throughout the history of mathematics. The most powerful contributions to a systematic investigation of polytopes in our century are due to Coxeter. In the last two decades, due to Coxeter's influence, particular attention has been paid to the combinatorial properties of regular figures. As a consequence the notion of a regular polytope has been extended in several directions.

Traditionally the cells of abstract polytopes were defined as images of convex polytopes under a particular type of mapping (see, for example, [15] and [21]) so that each cell was a topological ball. About 10 years ago Grünbaum suggested that it might be of interest to consider polytopes whose cells are images of toroidal maps. In [16] Grünbaum lists many interesting examples of these kinds of polytopes. Among them is a very interesting abstract polytope found independently by Coxeter and Shephard [12]. Further examples were discovered by Schulte [24], the author, and Colbourn (see [2] and [18]).

\footnotetext{
* Research supported by NSERC Canada Grant A8857.
} 
Grünbaum's notion of a polystroma, introduced in [16] as a special type of partially ordered set yielding a combinatorial structure that locally behaves like a polytope, provided a framework for the new approach to investigation of regular polytopes. The concept of a polystroma was later modified by Danzer [13] to become an (considerably more restrictive) incidence-polytope. Here we recall some definitions and notations. For details and the introduction to the theory of incidence-polytopes one should consult [14], [22], and [23].

An incidence-polytope $\mathscr{P}$ of dimension $n$, or briefly an $n$-incidence-polytope, is a partially ordered set with the properties (1)-(4) below.

(1) $\mathscr{P}$ has a smallest element $F_{-1}$ and a greatest element $F_{n}$.

(2) Every chain of $\mathscr{P}$ is contained in a maximal chain with exactly $n+2$ elements, a so-called flag.

The elements of $\mathscr{P}$ are called faces. Any face $F$ can be thought of as itself being a partially ordered set $\{G \mid G \leq F\}$ with elements of $\mathscr{P}$ majorized by $F$. The dimension of $F, \operatorname{dim} F$, can be defined as the number of faces of $\mathscr{P}$ in a flag of $\{G \mid G \leq F\}$ minus 2 . In particular, the dimension of $F_{n}$ is $n$, and faces of dimension 0,1 , and $(n-1)$ are called a vertex, an edge, and a cell, respectively. If $F$ is a vertex, the partially ordered set $\{G \mid G \geq F\}$ with elements of $\mathscr{P}$ minorized by $F$ is called a vertex-figure of a vertex $F$.

(3) If $f$ and $g$ are flags of $\mathscr{P}, f \neq g$, and $h=f \cap g$, then there is a finite sequence of flags $f=f_{1}, f_{2}, \ldots, f_{m}=g$, all containing $h$, such that $f_{i+1}$ differs from $f_{i}$ in exactly one face $(1 \leq i \leq m-1)$ (i.e., $\mathscr{P}$ is connected).

(4) For any two faces $F$ and $G$ with $F \leq G$ and $\operatorname{dim} F+1=m=\operatorname{dim} G+1$, there are exactly two faces $H$ with $\operatorname{dim} H=m$ such that $F \leq H \leq G$ $(m=0,1, \ldots, n-1)$.

If $\mathscr{P}^{*}$ is an incidence-polytope obtained from $\mathscr{P}$ by leaving the set of faces unchanged and replacing $\leq$ with $\geq$ it is called the dual of $\mathscr{P}$. $\mathscr{P}$ is said to be self-dual if $\mathscr{P}$ and $\mathscr{P}^{*}$ are isomorphic.

An incidence-polytope $\mathscr{P}$ is said to be regular if the group of its (order preserving) automorphisms is transitive on the flags of $\mathscr{P}$. If $\mathscr{P}$ is regular then its faces are regular and all faces of the same dimension are mutually isomorphic. Also, the vertex-figures of a regular incidence-polytope are regular and mutually isomorphic. This definition of regularity is quite restrictive, as there are many incidence-polytopes with groups that are transitive on the set of faces of any dimension but not transitive on the flags. Actually the incidence-polytopes we describe in Sections 4, 5, and 6 are 4-incidence-polytopes with groups transitive on faces (in some instances they are also transitive on flags).

One of the central problems in the theory of polytopes is the construction of finite incidence-polytopes with preassigned cells and vertex-figures. Of particular interest to us will be incidence-polytopes which Grünbaum refers to as naturally generated, meaning that they are built step-by-step from disjoint copies of cells identifying elements only as dictated by the vertex-figures. If all the cells are isomorphic to an $n$-incidence-polytope $\mathscr{C}$ and all the vertex figures are isomorphic 
to an $n$-incidence-polytope $\mathscr{V}$, then the naturally generated $(n+1)$ incidence-polytope is denoted by $\{\mathscr{C}, \mathscr{V}\}$. The two particular classes of naturally generated 4-incidence-polytopes mentioned in [16] are

$$
\mathscr{H}_{b, c}=\left\{\{6,3\}_{b, c},\{3,3\}\right\}
$$

and

$$
\mathscr{L}_{b, c}=\left\{\{4,4\}_{b, c},\{4,3\}\right\},
$$

where in both cases cells are isomorphic to toroidal maps (described in Section 2 ). These will be investigated in greater detail in Sections 4 and 5.

Finite twisted honeycombs, introduced by Coxeter [6], with "spherical" cells provided many interesting examples of incidence-polytopes (see [6], [9], and [10]). In Section 6 we extend this study by allowing cells to be toroidal.

\section{Maps of Type $\{4,4\}$ and $\{3,6\}$ on a Torus}

Any map on a torus with quadrangular faces four meeting at each vertex (of type $\{4,4\}$ ) can be derived from its universal covering $\{4,4\}$ (a tessellation of Euclidean plane by squares) by a suitable identification [11, p. 103]. The symmetry group $[4,4]$ of a regular plane tessellation by squares is generated by reflections $R_{\nu}$ in the sides of its characteristic triangle $[11$, p. 103] and has the presentation

$$
R_{\nu}^{2}=\left(R_{1} R_{2}\right)^{4}=\left(R_{2} R_{3}\right)^{4}=\left(R_{1} R_{3}\right)^{2}=1, \quad \nu=1,2,3 .
$$

The rotation subgroup $[4,4]^{+}$of the group in terms of the rotations $S=R_{2} R_{3}$ and $N=R_{3} R_{1}$ then has the following presentation

$$
S^{4}=N^{2}=(S N)^{4}
$$

(see p. 107 of [11], with $N=T$ ).

In the rotation group the translations $X=S N S$ and $Y=S^{2} N$ generate an abelian subgroup. Regarding $X$ and $Y$ as unit translations along the axis in the Cartesian coordinate system, the orbit of the point $(0,0)$ under $\langle X, Y\rangle$ (the group generated by $X$ and $Y$ ) is the set of vertices of $\{4,4\}$. The translations $X^{b} Y^{c}$ and $X^{-c} Y^{b}$ generate another subgroup of $[4,4]^{+}$(isomorphic to $\langle X, Y\rangle$ ). The square

$$
(b, c) \quad(0,0) \quad(-c, b) \quad(b-c, b+c)
$$

is the fundamental region for the group $\left\langle X^{b} Y^{c}, X^{-c} Y^{b}\right\rangle$. Identifying opposite edges of the square we obtain a map $\{4,4\}_{b, c}$ on a torus.

A map of type $\{4,4\}$ is said to be reflexible if its group is generated by three automorphisms satisfying (2.1) (and possibly some other relations). It is easy to see that $\{4,4\}$ is reflexible if and only if $b c(b-c)=0$. In this case $\{4,4\}_{b, c}$ is an instance of a regular 3-incidence-polytope. Now $X^{b} Y^{c}=1$ (or, equivalently, $\left.X^{-c} Y^{b}=1\right)$ if and only if

$$
(S N S)^{b}\left(S^{2} N\right)^{c}=1
$$


The groups $[4,4]_{b, 0}$ and $[4,4]_{c, c}$ of $\{4,4\}_{b, 0}$ and $\{4,4\}_{c, c}$, respectively, are given by (2.1) with the respective extra relations (derived from (2.3))

$$
\left(R_{1} R_{2} R_{3} R_{2}\right)^{b}=1 \quad \text { and } \quad\left(R_{3} R_{2} R_{1}\right)^{2 c}=1
$$

On the other hand, the group $[4,4]_{b, c}$ of a nonreflexible map $\{4,4\}_{b, c}$ is defined by relations (2.2) and (2.3). In this case the group, although transitive on faces of each dimension, is not transitive on the flags and $\{4,4\}_{b, c}$ is not a regular 3-incidence-polytope.

Maps $\{6,3\}_{b, c}$ and its dual $\{3,6\}_{b, c}$ are obtained similarly (for details see [18]). In both cases maps are reflexible if and only if $b c(b-c)=0$ in which case they are regular 3-incidence-polytopes. The groups of the reflexible maps $\{6,3\}_{b, 0}$ and $\{6,3\}_{c, c}$ and their duals are given by

$$
R_{\nu}^{2}=\left(R_{1} R_{2}\right)^{6}=\left(R_{2} R_{3}\right)^{3}=\left(R_{1} R_{3}\right)^{2}=1, \quad \nu=1,2,3 \text {, }
$$

with respective extra relations

$$
\left(R_{1} R_{2} R_{3}\right)^{2 b}=1 \quad \text { and } \quad\left(R_{3} R_{2} R_{1} R_{2} R_{1}\right)^{2 c}=1
$$

The group of a nonreflexible map $\{6,3\}_{b, c}$, and its dual, is given by

$$
S^{3}=N^{2}=(S N)^{6}=1
$$

together with the extra relation

$$
\left(S^{-1} N S N\right)^{b}\left(S N S^{-1} N\right)^{c}=1
$$

The nonreflexible maps $\{p, q\}_{b, c}$ and $\{p, q\}_{c, b}$ are considered to be the same by many authors although they are enantiomorphic (mirror images of each other) forms. For the purposes of this paper we will, in some instances, have to be careful in distinguishing between two different forms. Without loss of generality we may assume that $b, c \geq 0$.

\section{Hyperbolic Honeycombs and the Inversive Plane}

Some interesting examples of finite incidence-polytopes with toroidal cells can be derived from regular honeycombs in the three-dimensional hyperbolic space $\mathbf{H}^{3}$.

By a polyhedron we mean the union of plane polygons which form a topological 2-manifold without boundary and without self-intersections, where a plane polygon is a plane set bounded by finitely many line segments. We will, furthermore, assume that the plane polygons, called faces, are disjoint except for their edges. We use the Schläfli symbol $\{p, q\}$ to denote a regular polyhedron whose faces are regular $p$-gons, $q$ of which meet at any vertex. A three-dimensional honeycomb 
is a set of polyhedra, called cells, fitting together to fill all the space just once, so that every face of each polyhedron belongs to exactly one other polyhedron. A honeycomb is said to be regular if its cells are regular (polyhedra) and congruent. A regular honeycomb whose cells are $\{p, q\}$ 's, $r$ around each edge, is denoted by $\{p, q, r\}$ and is an instance of a 4-incidence-polytope. The arrangement of cells of a regular honeycomb $\{p, q, r\}$ around a common vertex corresponds to the arrangement of faces of polyhedron $\{q, r\}$ which is isomorphic to a vertex-figure of $\{p, q, r\}$.

There is only one regular three-dimensional honeycomb in the Euclidean space: $\{4,3,4\}$ is the space filling by cubes. The six regular four-dimensional polytopes are the only regular three-dimensional spherical honeycombs.

There are a total of 15 regular honeycombs in $\mathbb{H}^{3}:\{3,5,3\},\{5,3,4\},\{5,3,5\}$, $\{4,4,3\},\{4,4,4\},\{6,3,3\},\{6,3,4\},\{6,3,5\},\{6,3,6\},\{3,6,3\}$ and their duals. Of special interest to us will be regular honeycombs $\{p, q, r\}$ with $(p-2)(q-2)=4$ (so that $p$ is 6,3 , or 4 ). In each of these cases a cell of the honeycomb being a tessellated horosphere is isometric to a Euclidean tessellation [5, pp. 199-214].

Let us use Poincaré's half-space model for the hyperbolic space $\mathbf{H}^{3}$, and let $\Pi$ denote the absolute of $\mathrm{H}^{3}$. $\Pi$ being an extension of the Euclidean plane by the point at infinity can therefore be viewed as an inversive plane. It is now natural to establish a one-to-one correspondence between the planes of the hyperbolic space and the circles of the inversive plane (the intersections of planes of $\mathbb{H}^{3}$ by $\Pi$ ), and, consequently, the isomorphism between the group of reflections of $\mathrm{H}^{3}$ and the group of inversions in the inversive plane. For us it is important to notice that a group of displacements in $\mathrm{H}^{3}$ is isomorphic to a group of Möbius transformations. The above-mentioned isomorphism was first discovered by Liebmann [19].

The symmetry group $[p, q, r]$ of a honeycomb $\{p, q, r\}$ is generated by reflections $R_{\nu}$ in four planes $\rho_{\nu}$ which form an orthoscheme [7, p. 188] with dihedral angles $\Varangle\left(\rho_{1}, \rho_{2}\right)=\pi / p, \quad \Varangle\left(\rho_{2}, \rho_{3}\right)=\pi / q, \quad \Varangle\left(\rho_{3}, \rho_{4}\right)=\pi / r, \quad$ and $\quad \chi\left(\rho_{1}, \rho_{3}\right)=$ $\Varangle\left(\rho_{1}, \rho_{4}\right)=\Varangle\left(\rho_{2}, \rho_{4}\right)=\pi / 2$.

Since the absolute of the hyperbolic space $\mathbb{H}^{3}$ is isomorphic to the inversive plane, $[p, q, r]$ can be represented by a group generated by inversions in four circles, cutting one another at these same angles. The group $[p, q, r]$ in terms of the reflections $R_{\nu}$ has the following presentation

$$
\begin{array}{r}
R_{\nu}^{2}=\left(R_{1} R_{2}\right)^{p}=\left(R_{2} R_{3}\right)^{q}=\left(R_{3} R_{4}\right)^{r}=\left(R_{1} R_{3}\right)^{2}=\left(R_{2} R_{4}\right)^{2}=\left(R_{4} R_{1}\right)^{2}=1, \\
\nu=1,2,3,4 .
\end{array}
$$

We let $\omega$ denote the cube root of unity, so that it satisfies the equation $\omega^{2}+\omega+1=0$, and $i$ the fourth root of unity, i.e., $i^{2}+1=0$. As usual, $\tau$ will denote the "golden ratio" $(1+\sqrt{5}) / 2$ so that $\tau^{2}-\tau-1=0$.

$$
\begin{aligned}
& {[6,3,3]=\left\langle R_{1}(z)=\bar{z}, R_{2}(z)=-\bar{\omega} \bar{z}, R_{3}(z)=1-\bar{z}, R_{4}(z)=1 / \bar{z}\right\rangle} \\
& {[6,3,4]=\left\langle R_{1}(z)=\bar{z}, R_{2}(z)=-\bar{\omega} \bar{z}, R_{3}(z)=\sqrt{2}-\bar{z}, R_{4}(z)=1 / \bar{z}\right\rangle}
\end{aligned}
$$

See Fig. 1 . 


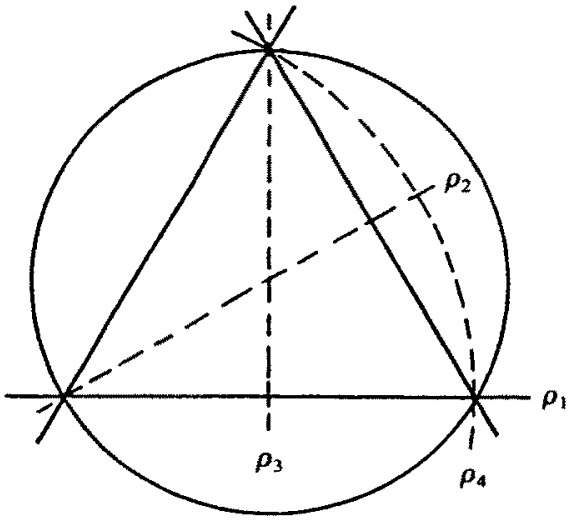

$[6,3,3]$

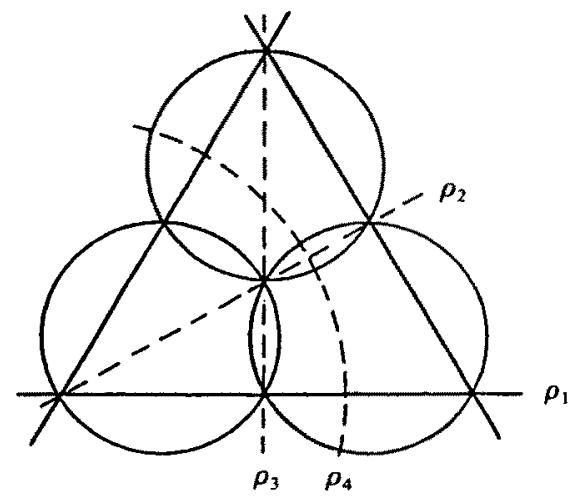

$[6,3,4]$

Fig. 1

$[6,3,5]=\left\langle R_{1}(z)=\bar{z}, R_{2}(z)=-\bar{\omega} \bar{z}, R_{3}(z)=\tau-\bar{z}, R_{4}(z)=1 / \bar{z}\right\rangle$, $[6,3,6]=\left\langle R_{1}(z)=\bar{z}, R_{2}(z)=-\bar{\omega} \bar{z}, R_{3}(z)=\sqrt{3}-\bar{z}, R_{4}(z)=1 / \bar{z}\right\rangle$.

See Fig. 2.

$$
\begin{aligned}
& {[3,6,3]=\left\langle R_{1}(z)=\bar{z}, R_{2}(z)=\omega \bar{z}, R_{3}(z)=1-\bar{z}, R_{4}(z)=1 / \bar{z}\right\rangle,} \\
& {[4,4,3]=\left\langle R_{1}(z)=\bar{z}, R_{2}(z)=i \bar{z}, R_{3}(z)=1-\bar{z}, R_{4}(z)=1 / \bar{z}\right\rangle,} \\
& {[4,4,4]=\left\langle R_{1}(z)=\bar{z}, R_{2}(z)=i \bar{z}, R_{3}(z)=\sqrt{2}-\bar{z}, R_{4}(z)=1 / \bar{z}\right\rangle .}
\end{aligned}
$$

See Fig. 3.

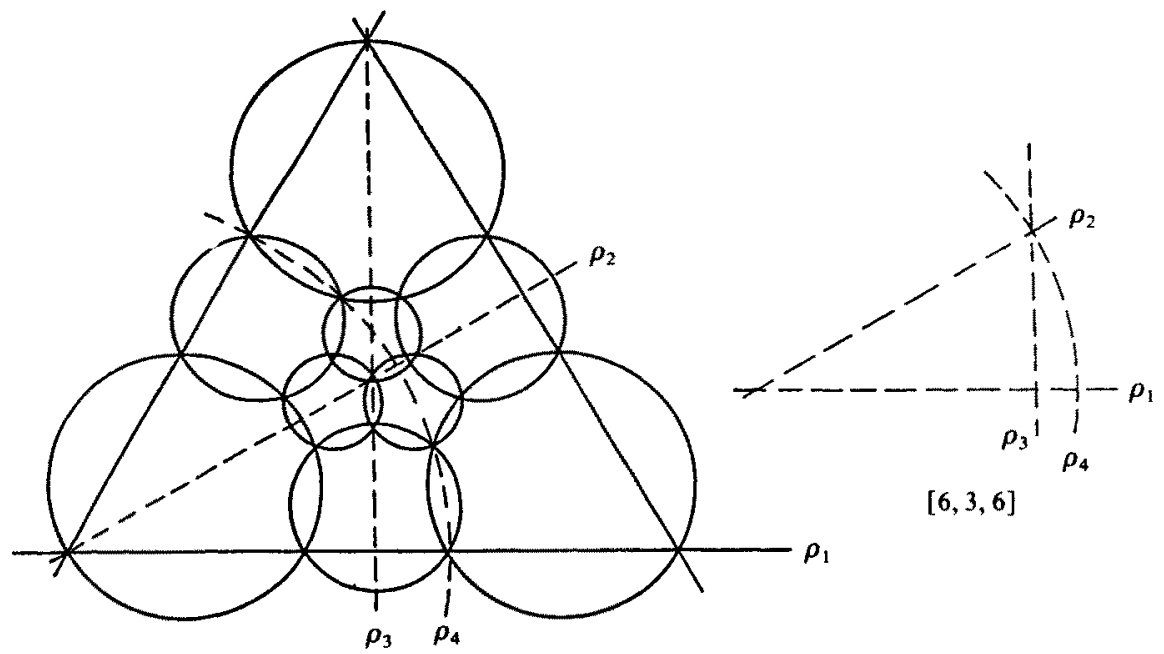

$[6,3,5]$

Fig. 2 


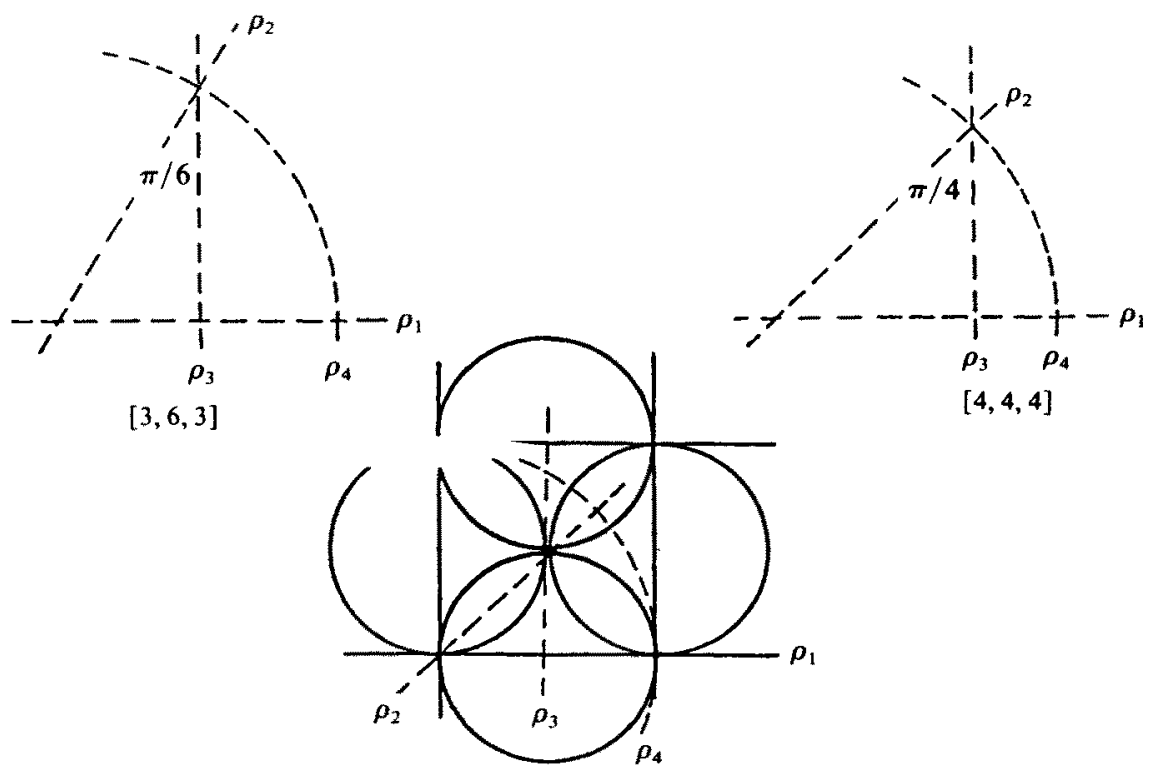

$[4,4,3]$

Fig. 3

In all these cases the inversions $R_{1}, R_{2}, R_{3}$ appear as reflections in lines, that is, in circles centered at infinity, and the lines of intersection of the corresponding planes in hyperbolic space are parallel, so that the centres of the cells of the honeycomb $\{p, q, r\}$ belong to the absolute. On the other hand, the same groups yield dual honeycombs whose vertices all belong to the absolute. The cells of the honeycombs with "ordinary" vertices, these being the orbit of the common point of the last three mirrors $R_{2}, R_{3}, R_{4}$, are inscribed in horospheres.

The cells of the honeycombs are represented by certain sets of circles, one circle for each face of the cell. But the vertices of the honeycomb have no nice analog in the inversive plane. (To be precise, a point of hyperbolic space is represented in the inversive plane by an elliptic bundle of circles.)

For the remaining three groups, $[3,5,3],[5,3,4]$, and $[5,3,5]$, expressions for the generating reflections do not all have as simple a form as the reflections above. We use an easy method of constructing pictures for finite polyhedral groups $\left[3\right.$, p. 523] to find three of the generating reflections $R_{\nu}$ :

$$
[3,5,3]=\left\langle R_{1}(z)=\bar{z}, R_{2}(z)=\omega \bar{z}, R_{3}(z)=z_{0}+\rho^{2} /\left(\bar{z}-z_{0}\right), R_{4}(z)=1 / \bar{z}\right),
$$

where $z_{0}$ and $\rho$ denote the centre and radius, respectively, of the circle of the inversion $R_{3} . \rho$ is a root of the equation $\tau^{-2} \rho^{2}-3 \rho+3=0$ and $z_{0}=3^{-1 / 2} \tau \rho$. The approximate values for $\rho$ are 6.678 and 1.176, and the approximate values for $z_{0}$ are 6.238 and 1.099 , respectively. 
Finally, for $p=4$ and $p=5$ we have

$$
[p, 3,5]=\left\langle R_{1}(z)=\bar{z}, R_{2}(z)=e^{i 2 \pi / 5} \bar{z}, R_{3}(z)=z_{0}+\rho^{2} /\left(\bar{z}-z_{0}\right), R_{4}(z)=1 / \bar{z}\right\rangle,
$$

where as before $z_{0}$ and $\rho$ are the center and radius, respectively, of the circle of the inversion $R_{3}$. When $p=4, \rho$ is a root of the equation $\rho^{2}-2 \tau \rho+2=0$ and $z_{0}=2^{-1 / 2} \rho$. The approximate values for $\rho$ are 2.404 and 0.832 , and the approximate values for $z_{0}$ are 1.700 and 0.588 , respectively. When $p=5, \rho$ is a root of the equation $\rho^{2}-(3 \tau+1) \rho+(\tau+2)=0$ and $z_{0}=5^{-1 / 4} \tau^{1 / 2} \rho$. In this case the approximate values for $\rho$ are 5.152 and 0.702 , and the approximate values for $z_{0}$ are 4.382 and 0.597 , respectively.

Of special interest to us will be to consider a rotation subgroup $[p, q, r]^{+}$of $[p, q, r]$. This group is generated by the three involutions (half-turns)

$$
L=R_{2} R_{4}, \quad M=R_{4} R_{1}, \quad N=R_{1} R_{3} .
$$

In terms of these generators $[p, q, r]^{+}$has the following presentation:

$$
L^{2}=M^{2}=N^{2}=(L M)^{p}=(L M N)^{q}=(M N)^{r}=1
$$

\section{Incidence-Polytopes with Cells of Type $\{4,4\}$}

We will now consider certain groups of $2 \times 2$ matrices derived in the following manner. Given a commutative ring $R$ and a subgroup $G$ of the units $R^{*}$ of $R$, one can consider the group

$$
\mathrm{SL}^{G}(2, R):=\left\{\left[\begin{array}{ll}
a & b \\
c & d
\end{array}\right] \mid a, b, c, d \in R \wedge a d-b c \in G\right\}
$$

and its quotient $\mathrm{PSL}^{G}(2, R)$ with respect to the center. In this and the following sections the representation by matrices is always meant in this sense.

Using the generating inversions for a group $[4,4, r]$, the three involutions $(3.2)$ can be represented by the Möbius transformations

$$
L(z)=i / z=-1 /(i z), \quad M(z)=1 / z=i /(i z), \quad N(z)=-z+x=(-i z+i x) / i
$$

with $x=1$ when $r=3$ and $x=\sqrt{2}$ when $r=4$. Since $\operatorname{det} L=i$ and $\operatorname{det} M=\operatorname{det} N=$ 1 , any transformation in the group $\langle L, M, N\rangle$ has a determinant equal to 1 or $i$. In matrix notation

$$
L=\left[\begin{array}{ll}
0 & i \\
1 & 0
\end{array}\right], \quad M=\left[\begin{array}{ll}
0 & 1 \\
1 & 0
\end{array}\right], \quad N=\left[\begin{array}{rr}
-1 & x \\
0 & 1
\end{array}\right]
$$


Each incidence-polytope $\left\{\{4,4\}_{b, c},\{4, r\}\right\}$ can now be derived from a hyperbolic honeycomb $\{4,4, r\}, r=4$ or 3 , by making suitable identifications. The relation (2.3) in terms of the involutions (3.2) can be written as

$$
(L M L M N)^{b}(L M N L M)^{c}=1 \text {. }
$$

The addition of (4.2) to (3.3) with $p=q=4$ gives us the presentation for the rotation subgroup of the group $\left[\{4,4\}_{b, c},\{4, r\}\right]$. If $b c(b-c) \neq 0$ this will be the whole group.

Since

$$
(L M L M N)^{b}(L M N L M)^{c}=\left[\begin{array}{cc}
1 & -x \\
0 & 1
\end{array}\right]^{b}\left[\begin{array}{cc}
1 & i x \\
0 & 1
\end{array}\right]^{c}=\left[\begin{array}{cc}
1 & x(c i-b) \\
0 & 1
\end{array}\right]
$$

the incidence-polytope $\left\{\{4,4\}_{b_{c} c},\{4, r\}\right\}$ is finite if and only if the factor group of the group generated by the matrices $(4.1)$ by the normal closure of (4.3) is finite.

The case with $r=3$ is particularly interesting. In this instance the subgroup of $\langle L, M, N\rangle$ generated by

$$
T=L M L=\left[\begin{array}{rr}
0 & 1 \\
-1 & 0
\end{array}\right], \quad U=N(L M)^{2}=\left[\begin{array}{ll}
1 & 1 \\
0 & 1
\end{array}\right], \quad V=L M N L M=\left[\begin{array}{ll}
1 & i \\
0 & 1
\end{array}\right]
$$

generates $\operatorname{PSL}(2, \mathbb{Z}[i])[1]$, where $\mathbb{Z}[i]$ denotes the ring of Gaussian integers (ring of polynomials in $i$ with integral coefficients). Obviously, $\langle T, U, V\rangle$ is a normal subgroup of $\langle L, M, N\rangle$ and hence the rotation group of $\{4,4,3\}$ is isomorphic to the semidirect product of $\operatorname{PSL}(2, \mathbb{Z}[i])$ with the cyclic group $C_{2}$ generated by $L$ :

$$
[4,4,3]^{+} \cong \operatorname{PSL}(2, \mathbb{Z}[i]) \cdot C_{2} \text {. }
$$

Consequently, the group of all $2 \times 2$ matrices of determinant equal to \pm 1 or $\pm i$ factored by its center is isomorphic to $\operatorname{PSL}(2, \mathbb{Z}[i]) \cdot C_{2}$.

To get the whole reflection group $[4,4,3]$ we adjoint one of the reflections $R_{\nu}$, say $R_{2}$, to $\langle L, M, N\rangle$. It is easy to check that $\langle T, U, V\rangle \cong \operatorname{PSL}(2, \mathbb{Z}[i])$ is a normal subgroup of $\left\langle R_{\nu}\right\rangle \cong[4,4,3]$ and since $\left\langle R_{2}, L\right\rangle=\left\langle R_{2}, R_{4}\right\rangle \cong D_{2}$ (the dihedral group of order 4$)$ and $\left(R_{2}, L\right\rangle \cap\langle T, U, V\rangle=1$ we have

$$
[4,4,3] \cong \operatorname{PSL}(2, \mathbb{Z}[i]) \cdot D_{2} \text {. }
$$

Hence, the group of the incidence-polytope $\mathscr{L}_{b, c}$ is the factor group of the group (4.6) or (4.5) by the normal closure of (4.3), with $x=1$, depending on whether $\mathscr{L}_{b, c}$ is reflexible or not. Consequently,

Theorem 1. $\mathscr{L}_{b, c}$ is finite if and only if the factor group of $\operatorname{PSL}(2, \mathbb{Z}[i])$ by the normal closure of

$$
\left[\begin{array}{ll}
1 & a \\
0 & 1
\end{array}\right]
$$

where $a=c i-b$, is finite. 
With the exception of $(b, c)$ equal to $(4,0)$ or $(5,0)$, whenever $b+c \leq 5, \mathscr{L}_{b, c}$ is known to be finite [2], [16], [12] and no other cases are known to be finite. An attempt was made to enumerate the cosets of $\left\langle R_{1}, R_{2}, R_{3}\right\rangle$ in $[4,4,3]$ using a computer but the computation exceeded the storage limits.

The polynomial $i^{2}+1=0$ is reducible in $\mathbb{Z}_{s}$ and its roots are \pm 2 . When $i=-2$, $b=1$, and $c=2$ we have $c i-b \equiv 0(\bmod 5)$ and the matrix (4.7) becomes the identity matrix. Since $i=-2$, det $L=2$ which is not a square in $\mathbb{Z}_{5}$. This, together with the fact that the matrices $T$ and $U$ from (4.4) generate the group of positive modular matrices (see, for example, p. 366 of [17]), implies that the matrices (4.1) generate PGL $(2,5)$ of order 120 . But the order of the group of $\mathscr{L}_{1,2}$ is 120 as well $[2$, p. 32$]$ and we conclude

$$
\left[\{4,4\}_{1,2},\{4,3\}\right] \cong \operatorname{PGL}(2,5) \cong S_{5} \text {. }
$$

In $\mathbb{Z}_{13}, i^{2}+1=0$ is reducible with roots \pm 5 . When $i=5, b=2$, and $c=3$ we have $c i-b \equiv 0(\bmod 13)$. With $i=5$, det $L=-5$ which is not a square in $\mathbb{Z}_{13}$ so that, as above, we conclude that in this case the matrices $(4.1)$ generate $\operatorname{PGL}(2,13)$ of order 2184. As above, considering that the order of the group of $\mathscr{L}_{2,3}$ is also $2184[2$, p. 32] we conclude

$$
\left[\{4,4\}_{2,3},\{4,3\}\right] \cong \operatorname{PGL}(2,13)
$$

The polynomial $i^{2}+1=0$ is also reducible in $\mathbb{Z}_{17}$ where its roots are \pm 4 . When $i=-4, b=1$, and $c=4$ we have $c i-b \equiv 0(\bmod 17)$ and the matrix (4.7) becomes the identity matrix. The matrices $(4.1)$ with $i=-4$ generate $\operatorname{PSL}(2,17)$ of order 2446 which is half of the order of $\left[\{4,4\}_{1,4},\{4,3\}\right]$, so that the group of $\mathscr{L}_{1,4}$ is an extension of $C_{2}$ by $\operatorname{PSL}(2,17)$.

When $b=1, c=3$, and $i=2$ we have $c i-b \equiv 0(\bmod 5)$ and $\operatorname{PGL}(2,5)$ is isomorphic to a quotient group of the group of $\mathscr{L}_{1,3}$ by a normal subgroup of order $6\left[2\right.$, p. 32]. The normal subgroup generated by $(L M L M N)^{5}$ and $(N L N L M)^{3}$ is the dihedral group $D_{3}$ of order 6 , and the group $\left[\{4,4\}_{1,3},\{4,3\}\right]$ is an extension of $\mathrm{D}_{3}$ by $\operatorname{PGL}(2,5)$.

The case with $r=4$ is interesting as well. In this instance the cells and the vertex-figures of the hyperbolic honeycomb are inscribed in horospheres and the honeycomb is self-dual. The group of the dual of $\{p, q, r\}$ is obtained from (3.1) interchanging $R_{1}$ with $R_{4}$ and $R_{2}$ with $R_{3}$. Consequently, the rotation group of the dual of $\{p, q, r\}$ is obtained from (3.3) by interchanging $L$ with $N$. From $\{4,4,4\}$ we can derive incidence-polytopes $\left\{\{4,4\}_{b, c},\{4,4\}_{d, e}\right\}$ with toroidal cells and toroidal vertex-figures. The presentation for the rotation subgroup of the group of this incidence-polytope is obtained by the addition of

$$
(N M N M L)^{d}(N M L N M)^{e}=1
$$

and (4.2) to (3.3) with $p=q=4$. When $b=d$ and $c=e$ the incidence-polytope is self-dual. 
We will now discuss several finite incidence-polytopes derived from $\{4,4,4\}$. For this purpose it will be convenient to represent the group $[4,4,4]$ by the following inversions:

$$
R_{1}(z)=\bar{z}, \quad R_{2}(z)=i \bar{z}, \quad R_{3}(z)=2-\bar{z}, \quad R_{4}(z)=2 / \bar{z} .
$$

Then the half-turns $L, M, N$ in matrix notation are

$$
L=\left[\begin{array}{cc}
0 & 2 \\
-i & 0
\end{array}\right], \quad M=\left[\begin{array}{ll}
0 & 2 \\
1 & 0
\end{array}\right], \quad N=\left[\begin{array}{rr}
-1 & 2 \\
0 & 1
\end{array}\right]
$$

and the relation (4.2) in terms of these matrices is (4.3) with $x=2$.

The incidence polytope $\left\{\{4,4\}_{1,2},\{4,4\}\right\}$ has 24 cells and six vertices. The addition of (4,2) with $b=1$ and $c=2$ to $[4,4,4]$ gives us the finite group of order 480 [2, p. 32]. In this group

$$
(N M N M L)^{2}(N M L N M)^{4}=1
$$

and the vertex-figures of the incidence-polytope are $\{4,4\}_{2,4}$. If, however, we add to the group the relation (4.8) with $d=1$ and $e=2$ we obtain a group of order 120 which is then the group of self-dual incidence-polytope $\left\{\{4,4\}_{1,2},\{4,4\}_{1,2}\right\}$.

In $\mathbb{Z}_{5}, i=-2$ is a root of $i^{2}+1=0$ and when $b=1$ and $c=2$ we have $c i-b \equiv 0$. Using (4.9) with $i=-2,(4.8)$ is satisfied when $d=1$ and $c=2$ and, furthermore,

$$
L M N L M=\left[\begin{array}{ll}
1 & 1 \\
0 & 1
\end{array}\right] \text { and } M L M=\left[\begin{array}{rr}
0 & 1 \\
-1 & 0
\end{array}\right](\bmod 5)
$$

Hence by $[17$, p. 366$]$, since det $M=-2$ which is not a square in $\mathbb{Z}_{5}$, the matrices (4.9) with $i=-2$ generate $\operatorname{PGL}(2,5)$ of order 120 . It follows that

$$
\left[\{4,4\}_{1,2},\{4,4\}_{1,2}\right] \cong \operatorname{PGL}(2,5)
$$

In terms of the above-mentioned matrices $y=(N M N M L)^{5}$ and $z=(N M L N M)^{5}$ are identity but in the group $\left[\{4,4\}_{1,2},\{4,4\}\right]$ they generate a normal subgroup isomorphic to the dihedral group $D_{2}$, so that the group $\left[\{4,4\}_{1,2},\{4,4\}\right]$ is an extension of $D_{2}$ by $\operatorname{PGL}(2,5)$.

The incidence-polytope $\left\{\{4,4\}_{2,3},\{4,4\}\right\}$ does not seem to be finite, but, however, the self-dual polytope $\left\{\{4,4\}_{2,3},\{4,4\}_{2,3}\right\}$ is finite and its group is of order 2184 suggesting that the group is $\operatorname{PGL}(2,13)$. We proceed to show that this is indeed true. Polynomial $i^{2}+1=0$ has solutions \pm 5 over $\mathbb{Z}_{13}$. With $b=2$ and $c=3, c i-b \equiv 0(\bmod 13)$ implies $i=5$. Matrices (4.9) with $i=5$ satisfy $(4.2)$ and (4.8) when $b=d=2$ and $c=e=3$. This matrix representation gives us

$$
U=(L M N L M)^{4}=\left[\begin{array}{ll}
1 & 1 \\
0 & 1
\end{array}\right] \text { and } U M U^{-2} M U=\left[\begin{array}{rr}
0 & 1 \\
-1 & 0
\end{array}\right](\bmod 13)
$$


and we conclude that

$$
\left[\{4,4\}_{2,3},\{4,4\}_{2,3}\right] \cong \operatorname{PGL}(2,13),
$$

since det $M=-2$ which is not a square in $\mathbb{Z}_{13}$.

If vertices of $\left\{\{4,4\}_{2,3},\{4,4\}\right\}$ were identified so that the vertex-figures were $\{4,4\}_{3,2}$ (mirror images of the cells) the honeycomb would collapse to a honeycomb with one cell only.

\section{Incidence-Polytopes with Cells of Type $\{6,3\}$ or $\{3,6\}$}

Using the generating inversions for a group $[6,3, r]$, the three involutions $(3.2)$ can be represented by the Möbius transformations

$$
L(z)=-\bar{\omega} / z=-\omega / \omega^{2} z, \quad M(z)=1 / z, \quad N(z)=-z+x
$$

with $x=1,2, \tau$ or $\sqrt{3}$ when $r=3,4,5$, or 6 , respectively. Since det $L=1$ and det $M=\operatorname{det} N=-1$, any transformation in the group $\langle L, M, N\rangle$ has a determinant equal to 1 or -1 . In matrix notation

$$
L=\left[\begin{array}{cc}
0 & -\omega \\
\omega^{2} & 0
\end{array}\right], \quad M=\left[\begin{array}{ll}
0 & 1 \\
1 & 0
\end{array}\right], \quad N=\left[\begin{array}{rr}
-1 & x \\
0 & 1
\end{array}\right] .
$$

Each incidence-polytope $\left\{\{6,3\}_{b, c},\{3, r\}\right\}$ can now be derived from the hyperbolic honeycomb $\{6,3, r\}$ by making suitable identifications. The relation (2.8) in terms of the involutions $L, M$, and $N$ can be written as

$$
(N M L N L M)^{b}(L M N M L N)^{\mathrm{c}}=1 .
$$

The addition of (5.2) to (3.3) with $p=6$ and $q=3$ gives us the presentation for the rotation subgroup of the group $\left[\{6,3\}_{b, c}\{3, r\}\right]$. If $b c(b-c) \neq 0$ this will be the entire group.

The honeycomb $\{6,3, r\}$ has finite vertex-figures when $r=3,4$, or 5 . When $r=6$ its vertex-figures are inscribed in horospheres and the honeycomb is self-dual. From $\{6,3,6\}$ we can derive incidence-polytopes $\left\{\{6,3\}_{b, c},\{3,6\}_{d, e}\right\}$ with toroidal cells and toroidal vertex-figures. The additional relation in this case is obtained from (5.2) by interchanging $L$ and $N$ (and $b, c$ changed to $d, e$, respectively) so that

$$
(L M N L N M)^{d}(N M L M N L)^{e}=1 .
$$

Since

$(N M L N L M)^{b}(L M N M L N)^{c}=\left[\begin{array}{cc}1 & -x \omega^{2} \\ 0 & 1\end{array}\right]^{b}\left[\begin{array}{cc}1 & x \omega \\ 0 & 1\end{array}\right]^{c}=\left[\begin{array}{cc}1 & x \omega(c-b \omega) \\ 0 & 1\end{array}\right]$

the incidence-polytope $\left\{\{6,3\}_{b, c},\{3, r\}\right\}$ is finite if and only if the factor group of the group generated by the matrices $(5.1)$ by the normal closure of $(5.3)$ is finite. 
The most interesting case is the one with $r=3$. This case is described in detail in [18]. When $r=3$ then $x=1$ and in this case matrices (5.1) generate the modular group $\operatorname{PSL}^{ \pm}(2, \mathbb{Z}[\omega]$ ): the group of all $2 \times 2$ matrices over $\mathbb{Z}[\omega]$ (ring of polynomials in $\omega$ with integral coefficients) with a determinant equal to 1 or -1 factored by its center. This group is isomorphic to the semidirect product of $\operatorname{PSL}(2, \mathbb{Z}[\omega])$ with the cyclic group $C_{2}$ generated by a matrix with a determinant equal to -1 :

$$
[6,3,3]^{+} \cong \operatorname{PSL}(2, \mathbb{Z}[\omega]) \cdot C_{2}
$$

Now, as in Section 4, we can show

$$
[6,3,3] \cong \operatorname{PSL}(2, \mathbb{Z}[\omega]) \cdot D_{2}
$$

and, as was essentially done in [18], we can conclude

Theorem 2. $\mathscr{K}_{b, c}$ is finite if and only if the factor group of $\operatorname{PSL}(2, \mathbb{Z}[\omega])$ by the normal closure of

$$
\left[\begin{array}{ll}
1 & a \\
0 & 1
\end{array}\right]
$$

where $a=c \omega-b \omega^{2}$, is finite.

Except for $(b, c)=(5,0)$, whenever $b+c \leq 5, \mathscr{H}_{h, c}$ is known to be finite (see [2], [16], and [18]) and there are no other known cases. Reference [18] can be consulted for an explicit recognition of the groups of these incidence-polytopes.

Theorems 1 and 2 are reminiscent of the situation where the factor group of the modular group $\operatorname{PSL}(2, \mathbb{Z})$ by the normal closure of the above matrix is finite if and only if $|a| \leq 5$, giving the usual polyhedral groups.

This can easily be seen as follows. The matrices

$$
S=\left[\begin{array}{ll}
1 & 1 \\
0 & 1
\end{array}\right] \text { and } T=\left[\begin{array}{rr}
0 & 1 \\
-1 & 0
\end{array}\right]
$$

generate the modular group $\operatorname{PSL}(2, \mathbb{Z})[11$, p. 94]. These generators satisfy the relations

$$
T^{2}=\left(S T^{3}\right)=1
$$

and, since the modular group is defined by the above relations [20, p. 108], it follows that $\operatorname{PSL}(2, \mathbb{Z})$ is isomorphic with the triangle group $(2,3, \infty)$. Factoring $\operatorname{PSL}(2, \mathbb{Z})$ by the normal closure of

$$
S^{a}=\left[\begin{array}{ll}
1 & a \\
0 & 1
\end{array}\right]
$$


results in a finite group if and only if the triangle group $(2,3, a)$ defined by

$$
S^{a}=T^{2}=(S T)^{3}=1
$$

is finite. On the other hand, $(2,3, a)$ is finite only when $a$ is 3,4 , or 5 giving tetrahedral, octahedral, or icosahedral groups, respectively (see, for example, $p$. 15 of [8]).

In group $[6,3,3]$ the four reflections $R_{1}, R_{2} R_{1} R_{2}, R_{3}$, and $R_{4}$ generate a subgroup which is isomorphic to $[3,6,3]$ and hence the Möbius transformations

$$
\begin{gathered}
l=R_{2} R_{1} R_{2} R_{4}=L M L=\left[\begin{array}{cc}
0 & \omega^{2} \\
\omega & 0
\end{array}\right], \quad m=R_{1} R_{4}=M=\left[\begin{array}{ll}
0 & 1 \\
1 & 0
\end{array}\right], \\
n=R_{1} R_{3}=N=\left[\begin{array}{rr}
-1 & 1 \\
0 & 1
\end{array}\right]
\end{gathered}
$$

generate a group isomorphic to $[3,6,3]^{+}$. The additional relation for $\{3,6\}_{b, c}$ yields, in terms of these matrices,

$$
(\operatorname{mln} \operatorname{lmn})^{b}(\operatorname{lmnmln})^{c}=\left[\begin{array}{cc}
1 & \omega^{2}-1 \\
0 & 1
\end{array}\right]^{b}\left[\begin{array}{cc}
1 & \omega-1 \\
0 & 1
\end{array}\right]^{c}=\left[\begin{array}{cc}
1 & c(\omega-1)+b\left(\omega^{2}-1\right) \\
0 & 1
\end{array}\right]
$$

Hence $\left\{\{3,6\}_{b, c},\{6,3\}\right\}$ is finite if and only if the subgroup of $\operatorname{PSL}^{ \pm}(2, \mathbb{Z}[\omega])$ generated by $l, m$, and $n$ factored by the normal closure of this matrix is finite.

\section{Twisted Honeycombs with Toroidal Cells}

Let $\{p, q, r\}$ be a regular hyperbolic honeycomb with generating reflections satisfying (3.1). The transformation $R_{1} R_{2} R_{3} R_{4}$ being conjugate to $R_{3} R_{4} R_{1} R_{2}=$ $R_{3} R_{1} \cdot R_{4} R_{2}$, which is the product of two half-turns about two nonintersecting edges of a characteristic orthoscheme, is a twist shifting a vertex of the honeycomb along an infinite polygon called a Petrie polygon [6, p. 25]. For a 4-incidencepolytope such polygons can be defined as a skew polygon such that every three consecutive edges, but not four, belong to a cell [10, p. 169].

Petrie polygons of regular incidence-polytopes are discussed in [24]. The group of the honeycomb is transitive on the set of all of its Petrie polygons. Conjugation by a reflection maps "right"-handed Petrie polygons onto "left"-handed ones and vice versa. In Twisted Honeycombs [6] Coxeter suggests identifying vertices of a honeycomb $\{p, q, r\}$ whenever the vertices are separated by $t$ steps along a "right"-handed Petrie polygon to obtain a twisted honeycomb $\{p, q, r\}_{1}$.

Assuming that the reflections $R_{i}$ were chosen so that $R_{1} R_{2} R_{3} R_{4}$ is indeed a "right"-handed twist, the addition of $\left(R_{1} R_{2} R_{3} R_{4}\right)^{\prime}=1$ to (3.1) gives us the group of $\{p, q, r\}_{r}$. In this case the left-handed twist $R_{4} R_{1} R_{2} R_{3}$ must have the same order $t$, so that the resulting twisted honeycomb is a reflexible twisted honeycomb which we will denote, as in [2], by $\{p, q, r\}_{t, t}$. This is an instance of a regular 4-incidence-polytope whose group we denote by $[p, q, r]$, 
On the other hand, considering only the rotation group $[p, q, r]^{+}$of the honeycomb generated by the half-turns (3.2) satisfying (3.3), the addition of

$$
(L N)^{\prime}=1
$$

to (3.3) will not necessarily result in a group of a regular incidence-polytope. The half-turn $M=R_{4} R_{1}$ transforms $R_{1} R_{2} R_{3} R_{4}$ into the "right"-handed twist $L N=R_{4} R_{2} R_{3} R_{1}$. The "left"-handed twist $L M N M=R_{2} R_{3} R_{4} R_{1}$ in this instance need not be of the same order $t$ as $L N$ and in fact need not be of a finite order. If the addition of (6.1) to (3.3) results in a finite group then let the order of LMNM be denoted by $t^{\prime}$.

If $t^{\prime}=t$ the resulting twisted honeycomb $\{p, q, r\}$, (which is the same as $\left.\{p, q, r\}_{t, t}\right)$ is a regular 4 -incidence-polytope with group $[p, q, r]_{r}$. If $t^{\prime} \neq t$ the resulting twisted honeycomb is not symmetric by reflections and hence not a regular incidence-polytope. These twisted honeycombs are called chiral and their group denoted by $((p, r, t ; q))$ is generated by the half-turns $L, M, N$ satisfying (3.3) and (6.1) (see pp. 125 and 142 of [4]).

Tables 1 and 2 lists all known twisted honeycombs with toroidal cells. Their duals are also twisted honeycombs and they have toroidal vertex-figures.

Using the matrix representations from Sections 4 and 5 we can in some instances explicitly recognize the groups of twisted honeycombs.

Matrices (5.1) with $x=1$, when $\omega=3$ generate $\operatorname{PSL}(2,13)$ and satisfy the defining relations for $((6,3,7 ; 3))$, and when $\omega=11$ generate $\operatorname{PSL}(2,37)$ and satisfy the defining relations for $((6,3,9 ; 3))$ :

$$
\begin{aligned}
& ((6,3,7 ; 3)) \cong \operatorname{PSL}(2,13) \\
& ((6,3,9 ; 3)) \cong \operatorname{PSL}(2,37)
\end{aligned}
$$

In the group $((6,3,6 ; 3))$ the transformation $(N M L N L M)^{2}(L M N M L N)$ of period 3 generates a normal subgroup $C_{3}$. The factor group is then isomorphic to $\left[\{6,3\}_{2,1},\{3,3\}\right] \cong \operatorname{PGL}(2,7)[18]$ and hence $((6,3,6 ; 3))$ is an extension of $C_{3}$ by $\operatorname{PGL}(2,7)$.

In the group $((6,3,8 ; 3))$ the transformation $(L M N M)^{6}$ of period 2 induces a normal subgroup $D_{2}$, the dihedral group of order 4 , whose generators we may take to be $(L M N M)^{6}$ and $(N M L M)^{6}$. I am grateful to J. Leech for pointing this out. The factor group is isomorphic to $((6,3,6 ; 3)$ ) (fix $L$ and $M$ and interchange $N$ with $M N M)$. Equivalently, in the group, $(N M L N L M)(L M N M L N)^{2}$ of period 2 induces a normal subgroup $A_{4}$, the alternating group of order 12 , whose generators we may take to be $(N M L N L M)(L M N M L N)^{2}$ and $(L M N M)^{6}$ so that $((6,3,8 ; 3))$ is an extension of $A_{4}$ by $\left[\{6,3\}_{1,2},\{3,3\}\right] \cong \operatorname{PGL}(2,7)$.

The above-mentioned isomorphism fixing $L$ and $M$ and interchanging $N$ with $M N M$ induces an isomorphism mapping $\left\{\{6,3\}_{b, c},\{3,3\}\right\}$ onto its mirror image $\left\{\{6,3\}_{c, b},\{3,3\}\right\}$, and mapping the "left"-handed twisted honeycomb onto the "right"-handed one (its mirror image). 


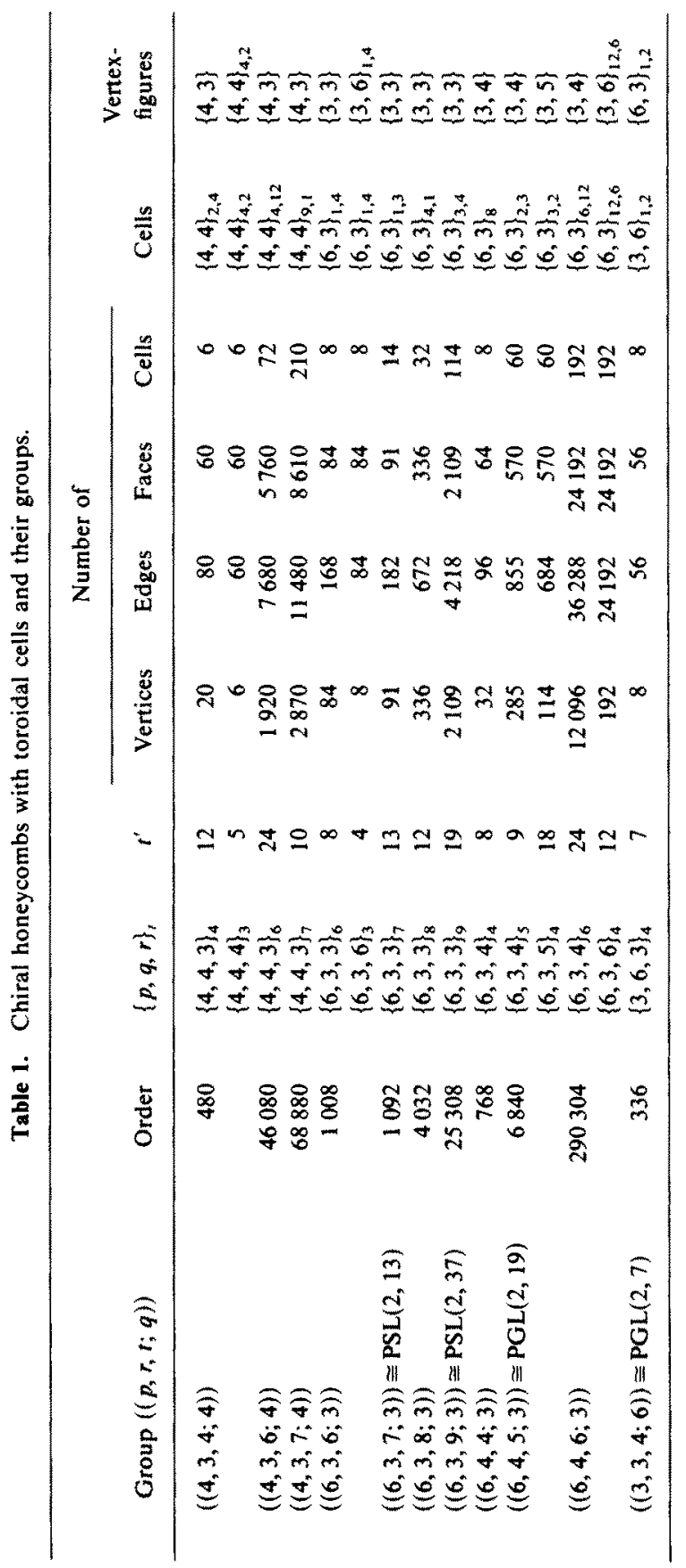




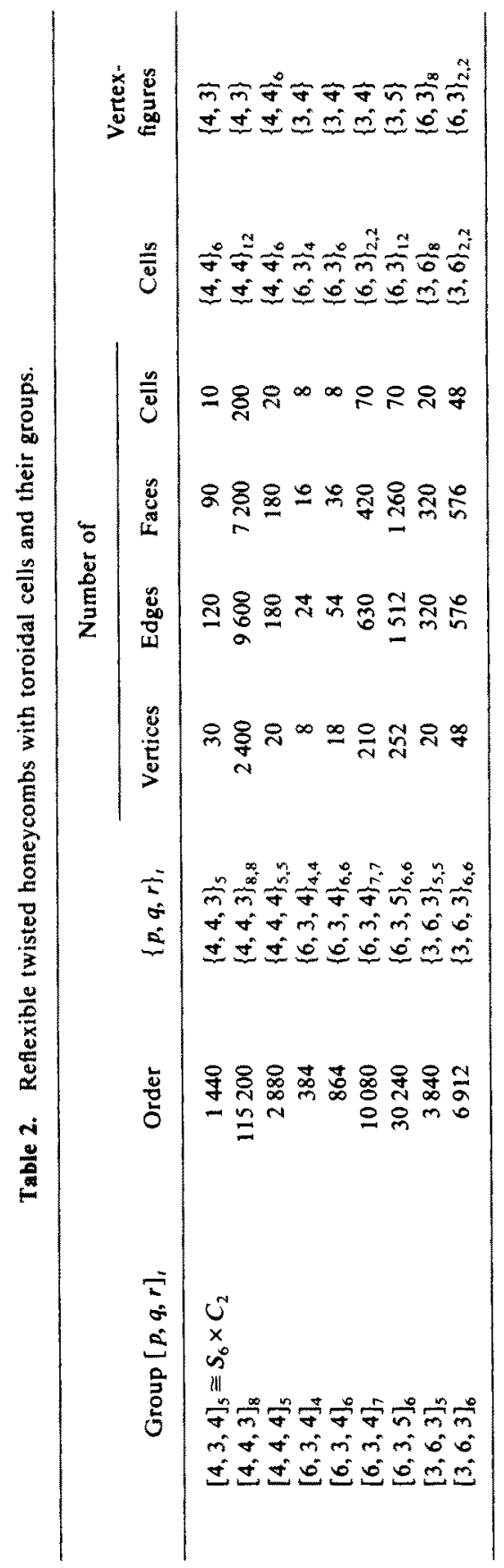


Matrices (5.4) with $\omega=-3$ generate $\operatorname{PGL}(2,7)$ and satisfy the defining relations for $((3,3,4 ; 6))$ and, since the orders are equal, we have

$$
((3,3,4 ; 6)) \cong \operatorname{PGL}(2,7) \text {. }
$$

In the group $\left[\{3,6\}_{1,2},\{6,3\}_{1,2}\right]$ the transformation $(L N)^{4}$ of order 2 generating a normal subgroup $C_{2}$ is in the center so that

$$
{ }_{1,2}[3,6,3]_{1,2} \cong \operatorname{PGL}(2,7) \times C_{2}
$$

In $[6$, p. 35] we can see that $((6,4,5 ; 3)) \cong((5,4,6 ; 3)) \cong \operatorname{PGL}(2,19)$. Another matrix representation can be derived from the matrices

$$
L=\left[\begin{array}{rr}
0 & -2 \\
\omega & 0
\end{array}\right], \quad M=\left[\begin{array}{ll}
0 & 2 \\
1 & 0
\end{array}\right], \quad N=\left[\begin{array}{rr}
-1 & 2 \\
0 & 1
\end{array}\right]
$$

(generating a group isomorphic to $[6,3,4]^{+}$, compare with (4.9)) by setting $\omega=-8$.

The group $[4,4,3]_{5}$, of the reflexible twisted honeycomb $\{4,4,3\}_{5}$, contains a central element $\left(R_{2} R_{1} R_{2} R_{3}\right)^{3}$ of order 2 . The addition of $\left(R_{2} R_{1} R_{2} R_{3}\right)^{3}=1$ to

$$
\begin{aligned}
& R_{\nu}^{2}=\left(R_{1} R_{2}\right)^{4}=\left(R_{2} R_{3}\right)^{4}=\left(R_{3} R_{4}\right)^{3}=\left(R_{1} R_{2} R_{3} R_{4}\right)^{5}=\left(R_{1} R_{3}\right)^{2}=\left(R_{1} R_{4}\right)^{2}=\left(R_{2} R_{4}\right)^{2} \\
& =1 \text {, } \\
& \nu=1,2,3,4 \text {, }
\end{aligned}
$$

results in the group isomorphic to the symmetric group $S_{6}[9, \mathrm{pp} .90-92]$, and hence

$$
[4,4,3]_{5} \cong S_{6} \times C_{2} \text {. }
$$

\section{References}

1. L. Bianchi, Geometrische Darstellung der Gruppen linearer Substitutionen mit ganzen complexen Coefficienten nebst Anwendungen auf die Zahlentheorie, Math. Ann. 38 (1891), 313-333.

2. C. J. Colbourn and A. Ivić Weiss, A census of regular 3-polystroma arising from honeycombs, Discrete Math. 50 (1984), 29-36.

3. H. S. M. Coxeter, An easy method for constructing polyhedral group-pictures, Amer. Math. Monthly 45 (1938), 522-525.

4. H. S. M. Coxeter, The abstract groups $G^{m_{n}, p}$, Trans. Amer. Math. Soc. 45 (1939), 73-150.

5. H. S. M. Coxeter, Twelve Geometric Essays, Southern Illinois University Press, Carbondale, IL, 1968.

6. H. S. M. Coxeter, Twisted Honeycombs, Regional Conference Series in Mathematics, No. 4, American Mathematical Society, Providence, R1, 1970.

7. H. S. M. Coxeter, Regular Polytopes, 3rd edn., Dover, New York, 1973.

8. H. S. M. Coxeter, Regular Complex Polytopes, Cambridge University Press, London, 1974.

9. H. S. M. Coxeter, Ten toroids and fifty-seven hemidodecahedra, Geom. Dedicata 13 (1982), 87-99.

10. H. S. M. Coxeter and A. Ivic Weiss, Twisted honeycombs $\{3,5,3\}$, and their groups, Geom. Dedicata 17 (1984), 169-179.

11. H. S. M. Coxeter and W. O. J. Moser, Generators and Relations for Discrete Groups, 4th edn., Springer-Verlag, Berlin, 1980. 
12. H. S. M. Coxeter and G. C. Shephard, Regular 3-complexes with toroidal cells, J. Combin. Theory Ser. B 22 (1977), 131-138.

13. L. Danzer, Regular incidence-complexes and dimensionally unbounded sequences of such, $I$, in Convexity and Graph Theory (M. Rosenfeld and J. Zaks, eds.), North Holland, Amsterdam, 1983.

14. L. Danzer and E. Schulte, Reguläre Incidenzkomplexe I, Geom. Dedicata 13 (1982), 295-308.

15. B. Grünbaum, Convex Polytopes, Wiley, London, 1967.

16. B. Grünbaum, Regularity of graphs, complexes and designs, in Problèmes Combinatoires et Théorie des Graphes, 191-197, Coll. Int. C.N.R.S. No. 260, C.N.R.S., Orsay, 1977.

17. L. K. Hua, Introduction to Number Theory, Springer-Verlag, Berlin, 1982.

18. A. Ivić Weiss, Incidence-polytopes of type $\{6,3,3\}$, Geom. Dedicata 20 (1986), 147-155.

19. H. Liebmann, Nichteuklidische Geometrie, Leipzig, 1905.

20. W. Magnus, Noneuclidean Tesselations and Their Groups, Academic Press, New York, 1974.

21. P. McMullen, Combinatorially regular polytopes, Mathematika 14 (1967), 142-150.

22. E. Schulte, Reguläre Incidenzkomplexe II, Geom. Dedicata 14 (1983), 33-56.

23. E. Schulte, Reguläre Incidenzkomplexe III, Geom. Dedicata 14 (1983), 57-79.

24. E. Schulte, Regular incidence-polytopes with euclidean or toroidal faces and vertex-figures, J. Combin. Theory Ser. A 40 (1985), 305-330.

Received April 23, 1986, and in revised form February 2, 1987. 